LTH 454

hep-ph/9907255

Revised Version

\title{
RG invariant solutions for the soft supersymmetry breaking parameters
}

\author{
I. Jack and D.R.T. Jones \\ Dept. of Mathematical Sciences, University of Liverpool, Liverpool L69 3BX, U.K.
}

\begin{abstract}
We show that the results for the soft couplings in supersymmetric gauge theories recently derived via the superconformal anomaly are equivalent to results obtained earlier from a consideration of exact RG-invariant trajectories. We give a full proof of these results for a general multi-coupling theory. Moreover, we demonstrate the existence of a distinct finite RG-invariant solution for the soft couplings in the case when the unbroken supersymmetric theory is finite.
\end{abstract}

July 1999 


\section{Introduction}

There has recently been interest in a specific and predictive source of supersymmetrybreaking originating in a vacuum expectation value for an auxiliary field in the supergravity multiplet, rather than from a hidden sector [1]. The result is that the various soft breaking masses and interactions (the gaugino mass $M$, the $\phi^{3}$ coupling $h^{i j k}$ and the $\phi \phi^{*}$ mass $m_{j}^{i}$ ) are all given, via the superconformal anomaly, in terms of the gravitino mass and the $\beta$-functions of the unbroken theory by simple relations that are renormalisation group invariant to all orders in perturbation theory. On the other hand, prior to this [2] [3], it was shown how to write down RG-invariant trajectories for the soft couplings, given RG-invariant trajectories for the Yukawa couplings. In this paper we shall show that this approach leads in fact to precisely the same results for the soft terms as the superconformal anomaly one, except that because there is no high-scale physics input, the gravitino mass is replaced by a constant of integration. We shall give a careful analysis demonstrating the RG-invariance of the results in the general multi-coupling case. We also demonstrate the existence of a distinct RG-invariant solution for the case when the unbroken supersymmetric theory is finite; corresponding to a class of all-orders finite theories with broken supersymmetry (this is not, of course, a class of conformal non-supersymmetric theories, because supersymmetry is broken by dimensionful interactions).

For a $N=1$ supersymmetric gauge theory with superpotential

$$
W(\Phi)=\frac{1}{6} Y^{i j k} \Phi_{i} \Phi_{j} \Phi_{k}+\frac{1}{2} \mu^{i j} \Phi_{i} \Phi_{j},
$$

we take the soft breaking Lagrangian $L_{S B}$ as follows:

$$
L_{\mathrm{SB}}=\left(m^{2}\right)_{i}^{j} \phi^{i} \phi_{j}+\left(\frac{1}{6} h^{i j k} \phi_{i} \phi_{j} \phi_{k}+\frac{1}{2} b^{i j} \phi_{i} \phi_{j}+\frac{1}{2} M \lambda \lambda+\text { h.c. }\right)
$$

We assume that there are no gauge-singlet fields and (for the time being) that $\mathcal{G}$ is simple.

In Refs. [2]-[5] (see also Ref. [6]) it was shown that the soft $\beta$-functions are given in terms of $\beta_{g}$ and the chiral supermultiplet anomalous dimension $\gamma^{i}{ }_{j}$ as follows:

$$
\begin{aligned}
\beta_{M} & =2 \mathcal{O}\left(\frac{\beta_{g}}{g}\right) \\
\beta_{h}^{i j k} & =\gamma^{(i}{ }_{l} h^{j k) l}-2 \gamma_{1}^{(i} Y^{j k) l} \\
\beta_{b}^{i j} & =\gamma^{(i}{ }_{l} b^{j) l}-2 \gamma_{1}^{(i}{ }_{l} \mu^{j) l} \\
\left(\beta_{m^{2}}\right)^{i}{ }_{j} & =\left(2 \mathcal{O} \mathcal{O}^{*}+2 M M^{*} g^{2} \frac{\partial}{\partial g^{2}}+\tilde{Y}_{l m n} \frac{\partial}{\partial Y_{l m n}}+\tilde{Y}^{l m n} \frac{\partial}{\partial Y^{l m n}}+X \frac{\partial}{\partial g}\right) \gamma^{i}{ }_{j}
\end{aligned}
$$


where we define $V^{(i j)}=V^{i j}+V^{j i}$, etc., and where

$$
\begin{gathered}
\left(\gamma_{1}\right)_{j}{ }_{j}=\mathcal{O} \gamma^{i}{ }_{j}, \\
\mathcal{O}=\left(M g^{2} \frac{\partial}{\partial g^{2}}-h^{l m n} \frac{\partial}{\partial Y^{l m n}}\right)
\end{gathered}
$$

and

$$
\tilde{Y}^{i j k}=Y^{l(j k}\left(m^{2}\right)^{i)}{ }_{l}
$$

A word on scheme dependence. The results given in Eq. (1.3) are valid in the DRED'

(or $\overline{\mathrm{DR}}^{\prime}$ ) scheme, introduced in Ref. [7] and established to all orders in Ref. [6]. They remain valid under a class of linked redefinitions of $g$ and $M[4]$ [5], and in particular in a scheme where the NSVZ form of the gauge $\beta$-function is valid.

\section{The conformal anomaly solution}

In a previous paper[2] we showed that on an RG trajectory $Y^{i j k}(g)$, the following relations are RG-invariant:

$$
\begin{aligned}
h^{i j k} & =-M g \frac{d Y^{i j k}}{d g}, \\
b^{i j} & =-M g \frac{d \mu^{i j}}{d g}, \\
\left(m^{2}\right)^{i}{ }_{j} & =\frac{g^{2}}{2 \beta_{g}}|M|^{2} \frac{d \gamma_{j}^{i}}{d g} .
\end{aligned}
$$

Remarkably, these relations can be supplemented by the following equation, which is also RG invariant:

$$
M=M_{0} \frac{\beta_{g}}{g},
$$

where $M_{0}$ is a constant. Presently we will verify the RG invariance of this equation; let us accept this for the moment, and substitute in Eq. (2.1). Noting that

$$
\beta_{Y}^{i j k}=\beta_{g} \frac{d Y^{i j k}}{d g}, \quad \beta_{\mu}^{i j}=\beta_{g} \frac{d \mu^{i j}}{d g},
$$

we obtain

$$
\begin{gathered}
h^{i j k}=-M_{0} \beta_{Y}^{i j k}, \\
b^{i j}=-M_{0} \beta_{\mu}^{i j}, \\
\left(m^{2}\right)^{i}{ }_{j}=\frac{1}{2}\left|M_{0}\right|^{2} \mu \frac{d \gamma^{i}{ }_{j}}{d \mu} .
\end{gathered}
$$


This is the form of the anomaly-generated relations as presented and applied in Ref. [1], except that $M_{0}$ becomes the gravitino mass, $m_{\frac{3}{2}}$. Several of the papers in Ref. [1] remark that Eqs. (2.2), (2.4) are in fact RG invariant; our purpose here is to establish this result to all orders in a general theory. It will be clear from our "bottom-up" approach that this is the unique RG-invariant integral of the equations that can be written without knowing the explicit form of the $\beta$-functions.

In Ref. [2] we demonstrated the RG invariance of Eqs. (2.1); we made, however, certain assumptions (which are spelt out later). In fact, these assumptions were not necessary, and it is possible to show that Eqs. (2.2) and (2.4) are RG-invariant for a completely general (non-finite) theory. Technical details are postponed to Appendix A, but we give the broad outlines here. The essential point is that the nature of the solution makes $\mathcal{O}$ a total derivative when acting on the relevant structures. If we substitute Eqs. (2.2) and (2.4a) in Eq. (1.5), we can show using Eq. (A.5) that

$$
\mathcal{O} \beta_{g}=\frac{1}{2} M_{0} \mu \frac{d}{d \mu} \beta_{g}
$$

and hence using Eq. (1.3a) we find immediately

$$
\beta_{M}=M_{0} \mu \frac{d}{d \mu}\left(\frac{\beta_{g}}{g}\right),
$$

which shows that Eq. (2.2) is RG-invariant for $M_{0}$ constant. (The RG invariance of this equation was actually demonstrated for a theory with no Yukawa couplings in Ref. [8].)

We can also show that (see Eq. (A.7))

$$
\mathcal{O} \gamma^{i}{ }_{j}=\frac{1}{2} M_{0} \mu \frac{d}{d \mu} \gamma^{i}{ }_{j}
$$

and hence, using Eqs. (1.4), (2.4a) in Eq. (1.3b), we obtain

$$
\beta_{h}^{i j k}=-M_{0}\left[\gamma^{(i} \beta_{Y}^{j k) l}+\mu \frac{d \gamma^{(i} l}{d \mu} Y^{j k) l}\right]=-M_{0} \mu \frac{d}{d \mu}\left[\gamma^{(i} Y^{j k) l}\right]=-M_{0} \mu \frac{d}{d \mu} \beta_{Y}^{i j k}
$$

demonstrating that Eq. $(2.4 a)$ is RG-invariant. The RG invariance of Eq. (2.4b) follows similarly. Finally, we can show that upon using Eqs. (2.4) in Eq. (1.3d), we just obtain

$$
\left(\beta_{m^{2}}\right)^{i}{ }_{j}=\frac{1}{2}\left|M_{0}\right|^{2}\left(\mu \frac{d}{d \mu}\right)^{2} \gamma_{j}^{i}
$$

which confirms the RG invariance of Eq. (2.4c). 
The authors of Ref. [3] derived results similar to our Eqs. (1.3), the major difference being that they omit the $X$-term in Eq. (1.3d), apparently believing that it would be absent given a supersymmetric regulator. Here we disagree; consider, for instance, a general NSVZ scheme. By this we mean one for which $\beta_{g}$ is given by [9]

$$
\beta_{g}^{\mathrm{NSVZ}}=\frac{g^{3}}{16 \pi^{2}}\left[\frac{Q-2 r^{-1} \operatorname{tr}[\gamma C(R)]}{1-2 g^{2} C(G)\left(16 \pi^{2}\right)^{-1}}\right],
$$

for which we showed[5] that $X$ is given by

$$
X^{\mathrm{NSVZ}}=-2 \frac{g^{3}}{16 \pi^{2}} \frac{S}{\left[1-2 g^{2} C(G)\left(16 \pi^{2}\right)^{-1}\right]},
$$

where

$$
Q=T(R)-3 C(G) \quad S=r^{-1} \operatorname{tr}\left[m^{2} C(R)\right]-M M^{*} C(G),
$$

and $r$ is the number of generators of the gauge group and $C(R)$ and $C(G)$ are the quadratic matter and adjoint Casimirs respectively. $X$ as given in Eq. (2.11) is clearly non-zero, and remains so in perturbatively related schemes such as $\overline{\mathrm{DR}}^{\prime}$; indeed, $X$ plays a crucial role in establishing the RG invariance of Eq. (2.4c) (see Eq. (A.10)). However, it is nevertheless possible, in special cases, to find a scheme in which $X$ vanishes. Consider a theory with $\gamma^{i}{ }_{j}$ and $\left(m^{2}\right)^{i}{ }_{j}$ diagonal and define a transformation from the NSVZ scheme to a new "holomorphic" scheme by

$$
\left.\frac{1}{\alpha^{\mathrm{H}}}=\frac{1}{\alpha^{\mathrm{NSVZ}}}+\frac{2}{16 \pi^{2}} C(G) \ln \alpha^{\mathrm{NSVZ}}-2 \frac{1}{16 \pi^{2}}\left[F(Y)+F\left(Y^{*}\right)\right]\right)
$$

(where $\alpha=g^{2}$ ), such that

$$
\sum_{a} R_{a i} \frac{\partial F}{\partial Y_{a}} Y_{a}=T\left(R_{i}\right)
$$

where $Y_{a}$ are the independent Yukawa couplings and $R_{a i}$ counts the number of fields $\Phi^{i}$ at the vertex $Y_{a}$. (This scheme, which was introduced in Ref. [5], is related but not identical to the usual holomorphic scheme, for a discussion of which see Refs. [10].) It is then easy to check using Eq. (2.10), (2.11), together with the transformation rule for $X$ given in Ref. [5], that $\beta_{\alpha^{H}}$ is one-loop exact and $X^{H}$ vanishes to all orders. As an example, if there at least as many independent Yukawa couplings than there are fields, then we may in general define a matrix $\tilde{R}_{i a}$ such that $\tilde{R}_{i a} R_{a j}=\delta_{i j}$. We may then take $F$ in Eq. (2.14) to be given by

$$
F\left(Y_{a}\right)=\sum_{i} T\left(R_{i}\right) \tilde{R}_{i a} \ln \left(Y_{a}\right)
$$


We end our discussion of the conformal anomaly case with a remark on the generalisation to a gauge group with a direct product structure $\left(G_{1} \otimes G_{2} \cdots\right)$. In fact, Eq. (2.4) is unaffected, but we must replace Eq. (2.2) by

$$
M_{I}=M_{0} \frac{\beta_{g_{I}}}{g_{I}} .
$$

Thus at a unification scale $M_{X}$ such that $g_{I}=g_{X}$ for all $I$, the gaugino masses do not themselves unify. Their ratios are, however, determined by the values of the corresponding gauge $\beta$-functions at $M_{X}$, that is, by the ratios of the one-loop $\beta$-function coefficients

(in the one-loop approximation). We note also that if one or more of the gauge group factors is abelian, then the Fayet-Iliopoulos $D$-term is renormalised, even if set zero at (say) $M_{X}$. If $D$ is eliminated using its equation of motion then this renormalisation gives rise to additional contributions to $\beta_{m^{2}}$ beyond those included in Eq. (1.3d); see for example Eq. (11) and Eq. (16) of Ref. [11]. Our results do, however, apply to the abelian case as long as we do not so eliminate $D$ ab initio. For a discussion of the phenomenological consequences of the linear D-term in the MSSM, see Ref. [12].

\section{The finite case}

We turn now to the finite case, corresponding to a choice of RG trajectories $Y^{i j k}(g)$ such that $\beta_{g}(g, Y(g))=\gamma(g, Y(g))=0$. Since in the NSVZ scheme $\gamma=0$ implies $\beta_{g}=0$ if $Q=0$, it is clear that there is a large class of $N=1$ theories of this type. Now in this case, Eqs. (2.2), (2.4) give zero for all the soft parameters. However, we know that there are non-trivial values for soft couplings consistent with finiteness, at least through two loops [11]; and we can in fact generalise this to all orders by demonstrating the existence of a distinct set of RG-invariant trajectories for this case. We begin by replacing Eq. (2.2) with

$$
M=M_{0},
$$

where $M_{0}$ is constant. Now from Eq. $(1.3 a, b)$ it is clear that to get $\beta_{M}=\beta_{h}=\beta_{b}=0$ we must have

$$
\mathcal{O}\left(\frac{\beta_{g}}{g}\right)=\mathcal{O} \gamma=0
$$

The solution to this is evidently

$$
\begin{aligned}
h^{i j k} & =-M_{0} g \frac{d Y^{i j k}}{d g} \\
b^{i j} & =\mathrm{const}
\end{aligned}
$$


(compare with Eq. (2.1a,b).) This was shown in Ref. [13, at least in the case of real couplings. The case of general couplings requires some additional assumptions, however. Once again denoting the independent Yukawa couplings by $Y_{a}$, we need the following identities:

$$
\begin{aligned}
Y_{a} \frac{\partial \gamma_{i}}{\partial Y_{a}} & =Y_{a}^{*} \frac{\partial \gamma_{i}}{\partial Y_{a}^{*}}, \quad(\text { no sum on } a) \\
Y_{a}^{\prime} \frac{\partial \gamma_{i}}{\partial Y_{a}} & =Y_{a}^{*} \frac{\partial \gamma_{i}}{\partial Y_{a}^{*}}, \quad(\text { no sum on } a) .
\end{aligned}
$$

Notice that in the non-finite case, Eq. (3.4b) follows from Eq. (A.7); but in the finite case Eq. (A.7) is trivial and we have been unable to establish either of Eq. (3.4a,b) for general couplings, though they are clearly true, for example, if $Y_{a}(g)$ can be chosen to be real. It follows from Eqs. $(\underline{3.4 b})$ and $(\underline{3.3 a})$ that

$$
\mathcal{O} \equiv \frac{1}{2} M_{0} g \frac{d}{d g},
$$

and hence we see from Eqs. (1.3a-c) that $\beta_{M}=\beta_{h}=\beta_{b}=0$.

Let us now turn to the case of $m^{2}$. It is clear that we must re-examine the derivation of Eq. (2.1d) in Ref. [2], since it is ill-defined in the finite case. This derivation proceeded by proposing a relation for $m^{2}$ of the form

$$
m_{i}^{2}=\frac{1}{3}\left|M_{0}\right|^{2} f_{i}(g)
$$

where we assume that $\left(m^{2}\right)^{i}{ }_{j}$ is diagonal and that $\left(m^{2}\right)^{i}{ }_{j}=m_{i}^{2} \delta^{i}{ }_{j}$. (In view of Eq. (1.3d), for consistency we must also assume $\gamma^{i}{ }_{j}=\gamma_{i} \delta^{i}{ }_{j}$.) We now need to ensure that when Eqs. (3.3a) and Eq. (3.6) are substituted into Eq. (1.3d), we obtain $\beta_{m_{i}^{2}}=0$. To prove this, we need an identity which follows from Eqs. (3.4.) These equations yield immediately

$$
\frac{Y_{a}^{\prime}}{Y_{a}}=\frac{Y_{a}^{\prime *}}{Y_{a}^{*}} .
$$

Acting on Eq. (3.4a) with $\frac{Y_{a}^{\prime} Y_{b}^{\prime}}{Y_{a}} \frac{\partial}{\partial Y_{b}}$, and using Eq. (3.7), we obtain

$$
Y_{a}^{\prime *} Y_{b}^{\prime} \frac{\partial^{2} \gamma_{i}}{\partial Y_{a}^{*} \partial Y_{b}}=Y_{a}^{\prime} Y_{b}^{\prime} \frac{\partial^{2} \gamma_{i}}{\partial Y_{a} \partial Y_{b}}+\frac{Y_{a}^{\prime 2}}{Y_{a}} \frac{\partial \gamma_{i}}{\partial Y_{a}}
$$

Using this identity, together with Eq. (3.4b), we can show (assuming that on the RG trajectory $\left.X=\left|M_{0}\right|^{2} \tilde{X}(g)\right)$ that

$$
\beta_{m_{i}^{2}}=\left|M_{0}\right|^{2}\left[\frac{1}{2} g^{2} \gamma_{i}^{\prime \prime}+\left(\frac{3}{2} g+\tilde{X}\right) \gamma_{i}^{\prime}\right]=0
$$


provided we choose

$$
\frac{1}{3} R_{a i} f_{i}=\left(\frac{3}{2} g+\tilde{X}\right) \frac{Y_{a}^{\prime}}{Y_{a}}+\frac{1}{2} g^{2}\left[\frac{Y_{a}^{\prime \prime}}{Y_{a}}-\left(\frac{Y_{a}^{\prime}}{Y_{a}}\right)^{2}\right], \quad(\text { no sum on } a)
$$

where $R_{a i}$ was defined earlier.

In our previous work we assumed $f_{i}(g)=f(g)$ for all $i$; this is sufficient but not necessary for an RG invariant solution[14]. The challenge is now to solve Eq. (3.10), a task which is complicated by the fact that $\tilde{X}$ depends on $f_{i}$, as can be seen from the explicit expression in the NSVZ scheme, Eq. (2.11).

In the finite case, the results of Ref. [3] were used (again omitting $X$ ) in Ref. [13] to derive a result equivalent to Eq. (3.10). However, even in the finite case there is no reason to expect $X$ to vanish in general. For example, consider the case of a finite trajectory for a single real Yukawa coupling $Y$ and a single chiral field. Suppose moreover that we are working in an NSVZ scheme as described above. The finite trajectory is given by $Y=a g+b g^{5}$ for some constants $a, b$ (there is no $O\left(g^{3}\right)$ term since one-loop finiteness automatically implies two-loop finiteness [15]). Then one can in principle solve Eqs. (3.10) and (2.11), and the result for $X$ is clearly non-zero at $O\left(g^{7}\right)$.

It is interesting to compare the leading order results for the scalar masses in the general (Eq. (2.4d)) and finite (Eq. (3.10)) cases. Neglecting Yukawa couplings, Eq. (2.4d) becomes

$$
\left(m^{2}\right)_{j}^{i}=-\frac{2 g^{4}}{\left(16 \pi^{2}\right)^{2}}\left|M_{0}\right|^{2} Q C(R)_{j}^{i}
$$

so that $m^{2}<0$ for a non-asymptotically free gauge group. This causes problems with slepton masses in the MSSM; various ways round this are discussed in Ref.[1]. On the other hand, Eq. (3.10) gives

$$
\sum_{i} R_{a i} m_{i}^{2}=\left|M_{0}\right|^{2}
$$

Finite GUT phenomenology attracts occasional interest [16] but is lacking a convincing derivation from an underlying theory. In the non-finite case, on the other hand, the conformal anomaly justification is very elegant[1], and is being pursued energetically in spite of the difficulty mentioned above. Unfortunately, all solutions proposed so far sully to some extent the purity of the conception.

In Appendix A we present some technical details associated with our derivation, and in Appendix B some remarks on the Method of Characteristics as applied to the $\beta$-function equations. 


\section{Appendix A.}

In Ref. [2], we demonstrated the RG invariance of Eqs. (2.1) in some detail for a theory with one real Yukawa coupling and a single superfield, and we also explained how to extend our results to the general case, under the special assumptions that $\gamma$ is diagonal and that Eq. (3.4a) holds. However, we can show that Eqs. (2.4) are RG-invariant for a completely general theory with arbitrary complex Yukawa couplings. The general strategy is as followed in Ref. [2] and sketched out above; if Eqs. (2.4) are applied, we can write the $\beta$-functions as given in Eqs. (1.3) in terms of total $\mu$-derivatives. However, in the general case we need a number of identities which are stated and proved here.

We start with the demonstration of RG invariance of Eq. (2.2). We define

$$
\mathcal{Q}=\sum_{k l m} \beta_{Y}^{k l m} \frac{\partial}{\partial Y^{k l m}}, \quad \mathcal{Q}^{*}=\sum_{k l m} \beta_{Y k l m} \frac{\partial}{\partial Y_{k l m}}
$$

Now on use of Eq. (2.4a), we find

$$
\mathcal{O}=\frac{1}{2} M_{0}\left(\beta_{g} \frac{\partial}{\partial g}+2 \mathcal{Q}\right)
$$

while

$$
\mu \frac{d}{d \mu}=\beta_{g} \frac{\partial}{\partial g}+\mathcal{R}
$$

where

$$
\mathcal{R}=\mathcal{Q}+\mathcal{Q}^{*}
$$

However, it is easy to show that

$$
\mathcal{Q}^{*} \beta_{g}=\mathcal{Q} \beta_{g}
$$

as follows: If we think in terms of the superfield diagrams contributing to $\beta_{g}$, then in view of Eq. (2.3), the effect of the operator on the left/right hand side of Eq. (A.5) is to give the sum of insertions of $\gamma$ onto each line emanating from each $Y / Y^{*}$ vertex, respectively. Each line emanating from a $Y$ vertex ends at $Y^{*}$ vertex, possibly after passing through some matter/gauge vertices. Since $\gamma$ commutes with the gauge generators, the effect of each operator is the same, namely to give the sum of insertions of a $\gamma$ onto each line joining a $Y$ and a $Y^{*}$ vertex. In view of Eq. (A.5), on comparing Eqs. (A.2), (A.3) we verify Eq. (2.5), enabling us to obtain Eq. (2.6). 
Turning now to $h^{i j k}$, the crucial step in the derivation of Eq. (2.8) was the use of Eq. (2.7). It is easy to see, using the same argument as for Eq. (A.5), that for any quantity $X^{i}{ }_{j}$ constructed according to supergraph Feynman rules, we have

$$
(\mathcal{Q} X)^{i}{ }_{j}-\left(\mathcal{Q}^{*} X\right)^{i}{ }_{j}=[\gamma, X]^{i}{ }_{j}
$$

Eq. (A.5) follows immediately, as does

$$
\mathcal{Q} \gamma=\mathcal{Q}^{*} \gamma
$$

whence Eq. (2.7) follows, leading immediately to Eq. (2.8).

To show the RG invariance of Eq. (2.4d), however, requires some care. The equation we wish to prove, Eq. (2.9), translates using Eq. (A.3) to

$$
\left(\beta_{m^{2}}\right)^{i}{ }_{j}=\frac{1}{2}\left|M_{0}\right|^{2}\left(\beta_{g} \frac{\partial}{\partial g}+\mathcal{R}\right)^{2} \gamma_{j}^{i}
$$

Two identities are required to prove this starting from Eq. (1.3d). The first (which follows by repeated application of Eq. (A.6)) is

$$
\mathcal{R}^{2} \gamma^{i}{ }_{j}=4 \beta_{Y}^{k l m} \beta_{Y p q r} \frac{\partial^{2}}{\partial Y^{k l m} \partial Y_{p q r}} \gamma^{i}{ }_{j}+(\mathcal{R} \gamma)^{\left({ }^{k}\right.}{ }_{n} Y^{l m) n} \frac{\partial \gamma^{i}{ }_{j}}{\partial Y^{k l m}}+(\mathcal{R} \gamma)^{n}{ }_{(k} Y_{l m) n} \frac{\partial \gamma^{i}{ }_{j}}{\partial Y_{k l m}} .
$$

The second identity is that if Eqs. $(2.4 a, b)$ are imposed, then

$$
\left|M_{0}\right|^{2} \mu \frac{d \beta_{g}}{d \mu}=3 \frac{\beta_{g}^{2}\left|M_{0}\right|^{2}}{g}+2 X
$$

An equivalent identity was deduced in Ref. [2] (Eq. (30), with $A=0$ ); we can also prove it directly using the explicit forms for $\beta_{g}$ and $X$ in the NSVZ scheme given in Eqs. (2.10), (2.11). Finally, expanding out $\mathcal{O O}^{*}$ in Eq. (1.3d), applying Eq. (2.4), and comparing with the result of expanding out the right-hand side of Eq. (A.8) and using Eqs. (A.9), (A.10), we find that Eq. (2.9) is satisfied.

\section{Appendix B.}

Here we indicate the relationship between the conformal anomaly solution given in Eqs. (2.2), (2.4) and the characteristics of Eqs. (1.3). For simplicity we restrict ourselves to the case of one real Yukawa coupling. Eqs. (1.3a,b) may be written as

$$
\begin{aligned}
\beta_{M^{\prime}} & =\left(M^{\prime} \frac{\partial}{\partial g}-h \frac{\partial}{\partial Y}\right) \beta_{g}, \\
\beta_{h} & =-\left(M^{\prime} \frac{\partial}{\partial g}-h \frac{\partial}{\partial Y}\right) \beta_{Y}
\end{aligned}
$$


where $M^{\prime}=g M$. Beginning with Eq. (B.1a), the gradients of the characteristics are given by

$$
\frac{1}{M_{0}} d t=\frac{d g}{M^{\prime}}=-\frac{d Y}{h}=\frac{1}{\beta_{M^{\prime}}} d \beta_{g}
$$

while Eq. (B.1b) gives

$$
\frac{1}{M_{0}} d t=\frac{d g}{M^{\prime}}=-\frac{d Y}{h}=-\frac{1}{\beta_{h}} d \beta_{Y}
$$

Together these yield:

$$
\begin{gathered}
M_{0} \frac{d g}{d t}=M^{\prime} \\
M_{0} \frac{d Y}{d t}=-h \\
M_{0} \frac{d \beta_{g}}{d t}=\beta_{M^{\prime}} \\
M_{0} \frac{d \beta_{Y}}{d t}=-\beta_{h}
\end{gathered}
$$

It can then be seen that requiring RG invariance of Eqs. (B.4a,b) leads to Eqs. (B.4c,d) and therefore Eqs. (B.4a,b) furnish RG-invariant expressions for $M^{\prime}$ and $h$. Indeed if we identify $t$ with $\mu$, then we regain Eqs. (2.2) and (2.4a). It is clear that Eq. (2.4b) will follow similarly. We have not pursued this approach with Eq. (1.3d) except to note that (in the real case) this second order PDE is parabolic, with characteristic gradient $\frac{d Y}{d g}=-h / M^{\prime}$, in common with Eqs. (B.1) above.

\section{Acknowledgements}

This work was supported in part by a Research Fellowship from the Leverhulme Trust. We thank Hugh Osborn for pointing out a couple of typographical errors in the original version. 


\section{References}

[1] L. Randall and R. Sundrum, hep-th/9810155;

G.F. Giudice, M.A. Luty, H. Murayama and R. Rattazzi, JHEP 9812 (1998) 27;

A. Pomarol and R. Rattazzi, hep-ph/9903448;

T. Gherghetta, G.F. Giudice, J.D. Wells, hep-ph/9904378;

Z. Chacko, M.A. Luty, I. Maksymyk and E. Ponton, hep-ph/9905390;

E. Katz, Y. Shadmi and Y. Shirman, hep-ph/9906296

[2] I. Jack, D.R.T. Jones and A. Pickering, Phys. Lett. B426 (1998) 73

[3] L.V. Avdeev, D.I. Kazakov and I.N. Kondrashuk, Nucl. Phys. B510 (1998) 289

[4] I. Jack and D.R.T. Jones, Phys. Lett. B415 (1997) 383

[5] I. Jack, D.R.T. Jones and A. Pickering, Phys. Lett. B432 (1998) 114

[6] N. Arkani-Hamed et al, Phys. Rev. D58 (1998) 115005

[7] I. Jack et al, Phys. Rev. D50 (1994) R5481

[8] J. Hisano and M. Shifman, Phys. Rev. D56 (1997) 5475

[9] V. Novikov et al, Nucl. Phys. B229 (1983) 381;

V. Novikov et al, Phys. Lett. B166 (1986) 329;

M. Shifman and A. Vainstein, Nucl. Phys. B277 (1986) 456

[10] N. Arkani-Hamed and H. Murayama, Phys. Rev. D57 (1998) 6638, hep-th/9707133

[11] I. Jack and D.R.T. Jones, Phys. Lett. B333 (1994) 372

[12] A. de Gouvêa, A. Friedland and H. Murayama Phys. Rev. D59 (1999) 095008

[13] D.I. Kazakov, Phys. Lett. B421 (1998) 211

[14] Y. Kawamura, T. Kobayashi and J. Kubo, Phys. Lett. B405 (1997) 64;

T. Kobayashi et al, Nucl. Phys. B511 (1998) 45;

T. Kobayashi, J. Kubo, and G. Zoupanos, Phys. Lett. B427 (1998) 291;

Y. Kawamura, T. Kobayashi and J. Kubo, Phys. Lett. B432 (1998) 108

[15] D.R.T. Jones and L. Mezincescu, Phys. Lett. B136 (1984) 242;

P. West, Phys. Lett. B136 (1984) 371;

A. Parkes and P. West, Phys. Lett. B138 (1984) 99;

D.R.T. Jones and L. Mezincescu, Phys. Lett. B138 (1984) 293

[16] D.R.T. Jones and S. Raby, Phys. Lett. B143 (1984) 137;

J. E. Bjorkman, D.R.T. Jones and S. Raby, Nucl. Phys. B259 (1985) 503;

J. Leon et al Phys. Lett. B156 (1985) 66;

A.V. Ermushev, D.I. Kazakov and O.V. Tarasov Nucl. Phys. B281 (1987) 72;

D. Kapetanakis, M. Mondragon and G. Zoupanos, Z. Phys. C60 (1993) 181;

N. G. Deshpande, Xiao-Gang He and E. Keith, Phys. Lett. B332 (1994) 88;

D.I. Kazakov et al Nucl. Phys. B471 (1996) 389;

J. Kubo and M. Mondragon and G. Zoupanos, Nucl.Phys.Proc.Suppl. 56B (1997) 281 